\title{
Kinematics analysis and simulation of 6R Robot Based on Matlab/Simulink
}

\author{
Jiwu Wang \\ School of Mechanical and Electronic Engineering, Beijing jiaotong University \\ Beijing, Haidian District, China \\ Shuo Han \\ School of Mechanical and Electronic Engineering, Beijing jiaotong University \\ Beijing, Haidian District, China \\ E-mail: jwwang@bjtu.edu.cn,19140566@bjtu.edu.cn \\ www.bjtu.edu.cn
}

\begin{abstract}
The $6 \mathrm{R}$ robot can imitate the human arm to complete some target grabbing tasks, so the kinematics analysis of the robot is significant in scientific research and practical application. In this paper, a kinematics solution method of $6 \mathrm{R}$ robot based on analytic method is introduced, which is faster and more accurate in solution than the numerical method. Then the trajectory of the end effector is planned by using the quintic polynomial method,in this way, there are no sudden changes in the speed of the end effector of the robot, and the operation is more stable.Furthermore, the accuracy of the kinematics solution method is verified and the motion trajectory of the manipulator is simulated by Matlab. At last ,the visualization of the robot kinematics model was realized based on the Simulink, and the kinematics simulation control system was established.
\end{abstract}

Keywords: 6-DOF robot ; forward kinematics ; inverse kinematics ; simulation.

\section{Introduction}

The industrial robot has played an important role in improving working conditions and production efficiency, and some algorithms and programs can control the robot to complete some specified tasks in a certain way.So that, the $6 \mathrm{R}$ robot is widely used in machining, electronic welding, industrial handling and other industries because of its flexibility and maneuver ability.

The basic characteristic of the $6 \mathrm{R}$ robot is the kinematics characteristic, so the kinematics analysis of the robot is significant in scientific research and practical application. In this paper,the mathematical model of the $6 \mathrm{R}$ robot is established by $\mathrm{D}-\mathrm{H}$ algorithm, and the forward and inverse kinematics calculation is completed,so that the angle of each joint can be found out when the robot grabs the target. Then the motion trajectory of the robot was simulated in the joint space, and the smooth curves of angular displacement,angular velocity were obtained.

Furthermore, the rationality of the D-H parameters and Inverse kinematics results of the robot were verified by MATLAB, and the visualization of the robot kinematics model was realized based on the Simulink, and the kinematics simulation control system was established.

\section{The Kinematics Analysis of the 6R Robot}




\subsection{Establish D-H coordinate system}

In this paper,the ABB-irb120 robot that shown in figure 1 is used as an example of $6 \mathrm{R}$ robot in our discussion.

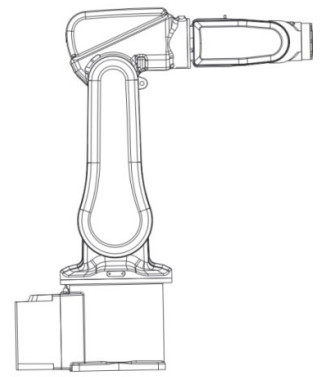

Fig. 1. The model of the $6 \mathrm{R}$ robot

We used the Denavit-Hartenberg (D-H) matrix representation for each link to describe the rotational and translational relationship between adjacent links. The coordinate system of each connected link is established, as shown in figure 2,and the D-H parameters were obtained, as shown in the table 1.

The coordinate system $\{0\}$ in figure 2 is a defined reference coordinate system, which is fixed on the base and usually coincides with the origin of the coordinate system $\{1\}$.

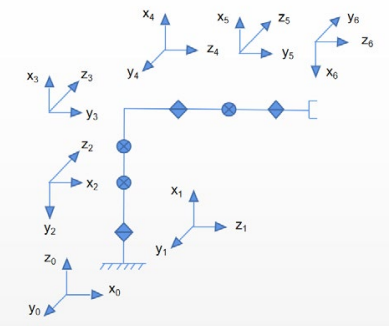

Fig. 2. Coordinate system of each link of the robot

Tab.1. D-H Parameter Table

\begin{tabular}{|c|c|c|c|c|}
\hline Joint $\mathrm{i}$ & $\theta_{\mathrm{i}}$ & $\alpha_{\mathrm{i}}$ & $\mathrm{a}_{\mathrm{i}}$ & $\mathrm{d}_{\mathrm{i}}$ \\
\hline 1 & $\theta_{1}$ & $-90^{\circ}$ & 0 & 290 \\
\hline 2 & $\theta_{2}$ & 0 & 270 & 0 \\
\hline 3 & $\theta_{3}$ & $-90^{\circ}$ & 70 & 0 \\
\hline 4 & $\theta_{4}$ & $90^{\circ}$ & 0 & 168 \\
\hline 5 & $\theta_{5}$ & $-90^{\circ}$ & 0 & 0 \\
\hline 6 & $\theta_{6}$ & 0 & 0 & 0 \\
\hline
\end{tabular}

\subsection{Forward Kinematics Solution}

The purpose of the forward kinematics is to find out the position and posture of the end effector that relative to the coordinate system $\{0\}$. The position and posture between adjacent coordinate systems are represented by $4 \times 4$ homogeneous transformation matrix as follows ${ }^{[1]}$ :

${ }^{\mathrm{I}-1} \mathrm{~T}_{\mathrm{i}}=\left[\begin{array}{cccc}\cos \theta_{\mathrm{i}} & -\sin \theta_{\mathrm{i}} \cos \alpha_{\mathrm{i}} & \sin \theta_{\mathrm{i}} \sin \alpha_{\mathrm{i}} & \operatorname{aicos} \theta_{\mathrm{i}} \\ \sin \theta_{\mathrm{i}} & \cos \theta_{\mathrm{i}} \cos \alpha_{\mathrm{i}} & -\cos \theta_{\mathrm{i}} \sin \alpha_{\mathrm{i}} & \operatorname{aisin} \theta_{\mathrm{i}} \\ 0 & \sin \alpha_{\mathrm{i}} & \cos \alpha_{\mathrm{i}} & \mathrm{d}_{\mathrm{i}} \\ 0 & 0 & 0 & 1\end{array}\right]$

${ }^{\mathrm{i}-1} \mathrm{~T}_{\mathrm{i}}$ represent the transformation matrix from 'the $\mathrm{i}-1$ coordinate system to the $\mathrm{i}$ coordinate system. Through substituting the D-H parameters in Table 1 into formula (1), the homogeneous transformation matrix between each connecting rod can be obtained as follows:

${ }^{0} \mathrm{~T}_{1}=\left[\begin{array}{cccc}\cos \theta_{1} & 0 & -\sin \theta_{1} & 0 \\ \sin \theta_{1} & 0 & \cos \theta_{1} & 0 \\ 0 & -1 & 0 & \mathrm{~d}_{1} \\ 0 & 0 & 0 & 1\end{array}\right]{ }^{1} \mathrm{~T}_{2}=\left[\begin{array}{cccc}\cos \theta_{2} & -\sin \theta_{2} & 0 & \mathrm{a}_{2} \cos \theta_{2} \\ \sin \theta_{2} & \cos \theta_{2} & 0 & \mathrm{a}_{2} \sin \theta_{2} \\ 0 & 0 & 1 & 0 \\ 0 & 0 & 0 & 1\end{array}\right]$ ${ }^{2} \mathrm{~T}_{3}=\left[\begin{array}{cccc}\cos \theta_{3} & 0 & -\sin \theta_{3} & \mathrm{a}_{3} \cos \theta_{3} \\ \sin \theta_{3} & 0 & \cos \theta_{3} & a_{3} \sin \theta_{3} \\ 0 & -1 & 0 & 0 \\ 0 & 0 & 0 & 1\end{array}\right]{ }^{3} \mathrm{~T}_{4}=\left[\begin{array}{cccc}\cos \theta_{4} & 0 & \sin \theta_{3} & 0 \\ \sin \theta_{4} & 0 & -\cos \theta_{3} & 0 \\ 0 & 1 & 0 & \mathrm{~d}_{4} \\ 0 & 0 & 0 & 1\end{array}\right]$ ${ }^{4} \mathrm{~T}_{5}=\left[\begin{array}{cccc}\cos \theta_{5} & 0 & -\sin \theta_{5} & 0 \\ \sin \theta_{5} & 0 & \cos \theta_{5} & 0 \\ 0 & -1 & 0 & 0 \\ 0 & 0 & 0 & 1\end{array}\right]{ }^{5} \mathrm{~T}_{6}=\left[\begin{array}{cccc}\cos \theta_{6} & -\sin \theta_{6} & 0 & 0 \\ \sin \theta_{6} & \cos \theta_{6} & 0 & 0 \\ 0 & 0 & 1 & 0 \\ 0 & 0 & 0 & 1\end{array}\right]$

So that,we can get the the spatial displacement and posture transformation of the 6th coordinate frame relative to the base/reference coordinate frame $\{0\}$ :

$$
{ }^{0} \mathrm{~T}_{6}={ }^{0} \mathrm{~T}_{1}{ }^{1} \mathrm{~T}_{2}{ }^{2} \mathrm{~T}_{3}{ }^{3} \mathrm{~T}_{4}{ }^{4} \mathrm{~T}_{5}{ }^{5} \mathrm{~T}_{6}
$$

\subsection{Inverse Kinematics Solution}

If the parameters of each joint and link of the robot and the relative position of the end effector relative to the fixed reference coordinate system are known, how to get the angle $\theta_{i}$ between the connected link of the robot, which is the inverse kinematics analysis solution.

Solution of $\theta_{1}$, According to the analysis of forward kinematics, we can multiply ${ }^{0} \mathrm{~T}_{1}{ }^{-1}$ form the left on both side of the Eq. (2) :

$$
{ }^{0} \mathrm{~T}_{1}{ }^{-1}{ }^{0} \mathrm{~T}_{6}={ }^{1} \mathrm{~T}_{2}{ }^{2} \mathrm{~T}_{3}{ }^{3} \mathrm{~T}_{4}{ }^{4} \mathrm{~T}_{5}{ }^{5} \mathrm{~T}_{6}
$$

By comparing the elements $(3,4)$ of the Eq.(3),we can know that :

$$
-\mathrm{p}_{\mathrm{y}} \mathrm{c}_{1}+\mathrm{p}_{\mathrm{x}} \mathrm{s}_{1}=0
$$


where $\mathrm{s}_{\mathrm{i}} \equiv \sin \theta_{\mathrm{i}}, \quad \mathrm{c}_{\mathrm{i}} \equiv \cos \theta_{\mathrm{i}}, \quad \mathrm{s}_{\mathrm{ij}} \equiv \sin \left(\theta_{\mathrm{i}}+\theta_{\mathrm{j}}\right), \quad \mathrm{c}_{\mathrm{ij}}$ $\equiv \cos \left(\theta_{\mathrm{i}}+\theta_{\mathrm{j}}\right)$, so that :

$$
\theta_{1}=\operatorname{atan} 2\left(p y-d_{6} a_{y}, p x-d_{6} a_{x}\right)
$$

Solution of $\theta_{2}$. We can multiply ${ }^{5} \mathrm{~T}_{6}{ }^{-1}$ form the left on both side of the Eq. (2) :

$$
{ }^{5} \mathrm{~T}_{6}{ }^{-1}{ }^{0} \mathrm{~T}_{6}={ }^{0} \mathrm{~T}_{1}{ }^{1} \mathrm{~T}_{2}{ }^{2} \mathrm{~T}_{3}{ }^{3} \mathrm{~T}_{4}{ }^{4} \mathrm{~T}_{5}
$$

By comparing the elements $(1,4),(2,4),(3,4)$ of the Eq.(6),we can know that :

$$
\begin{gathered}
\mathrm{p}_{5 \mathrm{x}}=\mathrm{p}_{\mathrm{x}}-\mathrm{d}_{6} \mathrm{a}_{\mathrm{x}}=\mathrm{c}_{1}\left(\mathrm{a}_{2} \mathrm{c}_{2}+\mathrm{a}_{3} \mathrm{c}_{23}-\mathrm{s}_{23} \mathrm{~d}_{4}\right) \\
\mathrm{p}_{5 \mathrm{y}}=\mathrm{p}_{\mathrm{y}}-\mathrm{d}_{6} \mathrm{a}_{\mathrm{y}}=\mathrm{s}_{1}\left(\mathrm{a}_{2} \mathrm{c}_{2}+\mathrm{a}_{3} \mathrm{c}_{23}-\mathrm{s}_{23} \mathrm{~d}_{4}\right) \\
\mathrm{p}_{5 \mathrm{z}}=\mathrm{p}_{\mathrm{z}}-\mathrm{d}_{6} \mathrm{a}_{\mathrm{z}}=\mathrm{d}_{1}-\mathrm{a}_{2} \mathrm{~s}_{2}-\mathrm{a}_{3} \mathrm{~s}_{23}-\mathrm{d}_{4} \mathrm{c}_{23}
\end{gathered}
$$

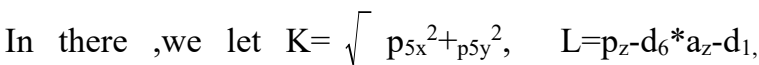
$\mathrm{M}=\left(\mathrm{L}^{2}+\mathrm{K}^{2}+\mathrm{a}_{2}{ }^{2}-\mathrm{a}_{3}{ }^{2}-\mathrm{d}_{4}{ }^{2}\right) / 2 \mathrm{a}_{2}$, according to the trigonometric theorem, $\theta_{2}$ can be obtained that ${ }^{[2]}$ :

$$
\theta_{2}=\operatorname{atan} 2(\mathrm{~K}, \mathrm{~L})-\operatorname{atan} 2\left(-\mathrm{M},\left(\mathrm{K}^{2}+\mathrm{L}^{2}-\mathrm{M}^{2}\right)^{0.5}\right)
$$

Solution of $\theta_{3}$. We can know that:

$$
\begin{aligned}
& \mathrm{K}-\mathrm{a}_{2 \mathrm{~s} 2}=\mathrm{a}_{3} \mathrm{~s}_{23}+\mathrm{d}_{4} \mathrm{c}_{23} ; \\
& \mathrm{L}-\mathrm{a}_{2} \mathrm{c}_{2}=\mathrm{a}_{3} \mathrm{c}_{23}+\mathrm{d}_{4} \mathrm{~s}_{23} ;
\end{aligned}
$$

according to the trigonometric theorem, $\theta_{23}$ can be obtained that:

$$
\begin{gathered}
\theta_{23}=\operatorname{atan} 2\left(a_{3}, d_{4}\right)-\operatorname{atan} 2\left(E,\left(a_{3}{ }^{2}+d_{4}{ }^{2}-E^{2}\right)^{0.5}\right) \\
E=K-a 2 * \cos \theta_{2} \\
\theta_{3}=\theta_{23}-\theta_{2}
\end{gathered}
$$

Solution of $\theta 4$. Multiply ${ }^{0} \mathrm{~T}_{3}{ }^{-1}$ form the left on both side of the Eq. (2) :

$$
\begin{gathered}
{ }^{0} \mathrm{~T}_{3}{ }^{-1}{ }^{0} \mathrm{~T}_{6=}{ }^{0} \mathrm{~T}_{1}{ }^{1} \mathrm{~T}_{2}{ }^{2} \mathrm{~T}_{3} \\
\mathrm{~W}=-\mathrm{s}_{4} \mathrm{~S}_{5}=\mathrm{a}_{\mathrm{x}} \mathrm{s}_{1}-\mathrm{a}_{\mathrm{y}} \mathrm{c}_{1} \\
\mathrm{Z}=-\mathrm{c}_{4} \mathrm{~S}_{5}=\mathrm{c}_{1} \mathrm{c}_{23} \mathrm{a}_{\mathrm{x}}-\mathrm{s}_{23} \mathrm{a}_{\mathrm{z}}+\mathrm{s}_{1} \mathrm{c}_{23} \mathrm{a}_{\mathrm{y}}
\end{gathered}
$$

So that,we can get the angle $\theta_{4}$ :

$$
\theta_{4}=\operatorname{atan} 2(\mathrm{~W}, \mathrm{Z}) \text {; }
$$

Solution of $\theta_{5}$. Multiply ${ }^{0} \mathrm{~T}_{4}{ }^{-1}$ form the left on both side of the Eq. (2)

$$
{ }^{0} \mathrm{~T}_{4}{ }^{-1}{ }^{0} \mathrm{~T}_{6}={ }^{4} \mathrm{~T}_{5}{ }^{5} \mathrm{~T}_{6}
$$

By comparing the elements of the Eq.(11),we can know that :

$$
\begin{gathered}
\mathrm{s}_{5}=-\mathrm{a}_{\mathrm{x}}\left(\mathrm{s}_{1} \mathrm{~s}_{4}+\mathrm{c}_{1} \mathrm{c}_{4} \mathrm{c}_{23}\right)-\mathrm{a}_{\mathrm{y}}\left(\mathrm{c}_{1} \mathrm{~S}_{4}-\mathrm{s}_{1} \mathrm{c}_{4} \mathrm{c}_{23}\right)-\mathrm{a}_{\mathrm{z}} \mathrm{c}_{4} \mathrm{~s}_{23} \\
\mathrm{c}_{5}=-\mathrm{a}_{\mathrm{x}} \mathrm{c}_{1} \mathrm{~s}_{23}-\mathrm{a}_{\mathrm{y}} \mathrm{s}_{1} \mathrm{~s}_{23}-\mathrm{a}_{\mathrm{z}} \mathrm{c}_{23} \\
\theta_{5}=\operatorname{atan} 2\left(\mathrm{~s}_{5}, \mathrm{c}_{5}\right)
\end{gathered}
$$

Solution of $\theta_{6}$. It is similar to the solution of $\theta_{5}$,we just need to multiply ${ }^{0} \mathrm{~T}_{5}{ }^{-1}$ form the left on both side of the Eq. (2)

$$
{ }^{0} \mathrm{~T}_{5}{ }^{-1}{ }^{0} \mathrm{~T}_{6=}{ }^{5} \mathrm{~T}_{6}
$$

By comparing the elements of the Eq.(13),we can know that :

$$
\begin{gathered}
\mathrm{s}_{6}=-\mathrm{n}_{\mathrm{x}}\left(\mathrm{s}_{1} \mathrm{c}_{4}+\mathrm{c}_{1} \mathrm{~S}_{4} \mathrm{c}_{23}\right)-\mathrm{n}_{\mathrm{y}}\left(\mathrm{c}_{1} \mathrm{c}_{4}+\mathrm{s}_{1} \mathrm{~S}_{4} \mathrm{c}_{23}\right)+\mathrm{n}_{\mathrm{z}} \mathrm{s}_{4} \mathrm{~S}_{23} \\
\mathrm{c}_{6}=\mathrm{o}_{\mathrm{x}}\left(\mathrm{c}_{4} \mathrm{~S}_{1}-\mathrm{c}_{1} \mathrm{~S}_{4} \mathrm{c}_{23}\right)-\mathrm{o}_{\mathrm{y}}\left(\mathrm{c}_{1} \mathrm{c}_{4}+\mathrm{s}_{1} \mathrm{~S}_{4} \mathrm{c}_{23}\right)+\mathrm{o}_{\mathrm{z}} \mathrm{S}_{4} \mathrm{~S}_{23} \\
\theta_{6}=\operatorname{atan} 2\left(\mathrm{~s}_{6}, \mathrm{c}_{6}\right)
\end{gathered}
$$

\subsection{Trajectory planning of the $6 R$ robot}

In this paper, we use the method of quintic polynomial interpolation to plan the trajectory of the $6 \mathrm{R}$ robot.

$$
\theta(t)=a_{0}+a_{1} t+a_{2} t^{2}+a_{3} t^{3}+a_{4} t^{4}+a_{5} t^{5}
$$

So that, the function expression of the velocity is:

$$
\theta(t)^{\prime}=a_{1}+2 a_{2} t+3 a_{3} t^{2}+4 a_{4} t^{3}+5 a_{5} t^{4}
$$

And the function expression of the acceleration is:

$$
\theta(\mathrm{t}) "=2 \mathrm{a}_{2}+6_{\mathrm{a} 3} \mathrm{t}+12 \mathrm{a}_{4} \mathrm{t}^{2}+20 \mathrm{a}_{5} \mathrm{t}^{3}
$$

By making constraints about the position, angular velocity and angular acceleration of each joint in space at the starting and ending positions of the path point:

$$
\left\{\begin{array}{l}
\theta\left(t_{0}\right)=a_{0} \\
\theta\left(t_{f}\right)=a_{0}+a_{1} t_{f}+a_{2} t_{f}{ }^{2}+a_{3} t_{f}{ }^{3}+a_{4} t_{f}{ }^{4}+a_{s} t_{f}{ }^{5} \\
\dot{\theta}\left(t_{0}\right)=a_{1} \\
\dot{\theta}\left(t_{f}\right)=a_{1}+2 a_{2} t_{f}+3 a_{3} t_{f}{ }^{2}+4 a_{4} t_{f}{ }^{3}+5 a_{5} t_{f}{ }^{4} \\
\ddot{\theta}\left(t_{0}\right)=2 a_{2} \\
\ddot{\theta}\left(t_{f}\right)=2 a_{2}+6 a_{3} t_{f}+12 a_{4} t_{f}{ }^{2}+20 a_{5} t_{f}{ }^{3}
\end{array}\right.
$$

So this is obtained from the above formula:

$$
\left\{\begin{array}{l}
a_{0}=\theta_{0} \\
a_{1}=\dot{\theta}_{0} \\
a_{2}=\frac{\ddot{\theta}_{0}}{2} \\
a_{3}=\frac{20 \theta_{f}-20 \theta_{0}-\left(8 \dot{\theta}_{f}+12 \dot{\theta}_{0}\right) t_{f}-\left(3 \ddot{\theta}_{0}-\ddot{\theta}_{f}\right) t_{f}^{2}}{2 t_{f}^{3}} \\
a_{4}=\frac{30 \theta_{f}-30 \theta_{0}-\left(14 \dot{\theta}_{f}+16 \dot{\theta}_{0}\right) t_{f}+\left(3 \ddot{\theta}_{0}-2 \ddot{\theta}_{f}\right) t_{f}^{2}}{2 t_{f}^{3}} \\
a_{s}=\frac{12 \theta_{f}-12 \theta_{0}-\left(6 \dot{\theta}_{f}+6 \dot{\theta}_{0}\right) t_{f}-\left(\ddot{\theta}_{0}-\ddot{\theta}_{f}\right) t_{f}^{2}}{2 t_{f}^{3}}
\end{array}\right.
$$

By substituting the above coefficients into the basic expression, the function expression using quintic polynomial interpolation method can be obtained.

(C) The 2020 International Conference on Artificial Life and Robotics (ICAROB2020), Jan. 9-12, B-Con Plaza, Beppu, Oita, Japan 


\section{The Kinematics Simulation Verification based on MATLAB}

We use the Matlab Robotics Toolbox to verify the accuracy of kinematics solution. By given a joint angle group ' $\mathrm{q}=\left[\mathrm{pi} / 3, \mathrm{pi} / 4,3^{*} \mathrm{pi} / 4,-\mathrm{pi} / 5, \mathrm{pi} / 5, \mathrm{pi} / 6\right]$ ', we use the forward and inverse kinematics algorithm finished in chapter 2.2 and 2.3 to get the pose and posture matrix of end effector, and the solution of each joint angle in Matlab, as shown in figure 3.

Then by comparing with the result that obtained by using the 'Fkine' and 'Ikine' function of Matlab,we found that the two results are consistent,so that the kinematics solution is verified.

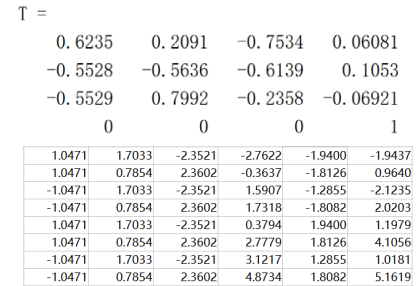

Fig. 3. Kinematics solution result finished by Matlab

The trajectory planning of the robot is also simulated by Matlab ,the curve of the velocity and acceleration of each joint angle with time, shown in the figure 4.

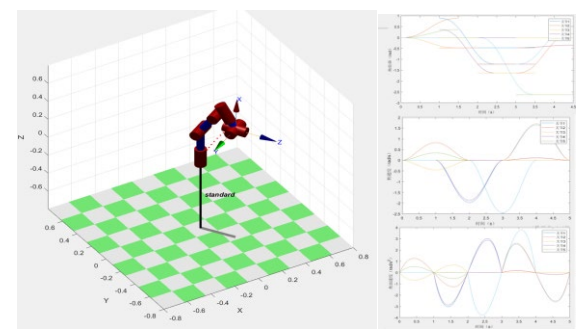

Fig. 4. Trajectory path and joint motion characteristic curve

\section{The Visual simulation based on Simulink}

The robot is modeled by SolidWorks 2016. First, the models of the robot is built and assembled. Then the assembly model is converted into the format of .urdf and imported into the Simulink. Then by using the modular simulation interface of the Simulink and referring with the previous solving function, the robotic visual simulation framework is finished,as shown in figure 5 and figure 6.

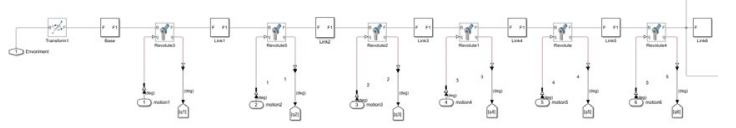

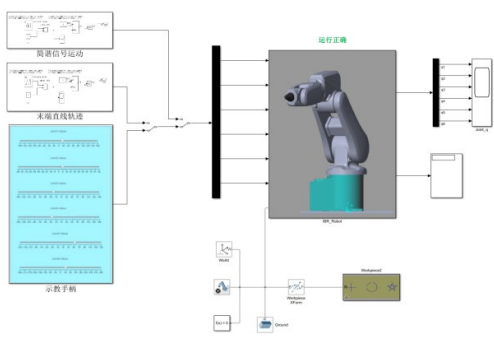

Fig. 6. Kinematics visual simulation by Simulink

\section{Conclusion}

In this paper,we take the IRB120 robot as the research object, using the D-H method to establish the connecting rod coordinate system, finish the solution of the forward and inverse kinematics analysis, get the $6 \mathrm{R}$ robot kinematics algorithm. Then the Matlab is used to model and simulate the robot. It can be seen from the simulation results that the model of the robot established in this study is correct, and the trajectory planning of the end effector is carried out, and the change of the angle of each joint with time is recorded, which verified the effectiveness of the kinematics algorithm.

\section{Acknowledgements}

This work was supported by the Key Research and Development Program (No.M18GY300021).

\section{References}

1. Guan Wei, "Research on obstacle avoidance path and trajectory planning foe six-dof manipulator," D. Shanghai Jiao Tong University, Jan.2016.

2. Hu Yaqing, $\mathrm{Yu}$ Jinpeng, Kinematics Analysis and Modeling Simulation Based on ABB-IRB120 Robot.J.Journal of Qingdao University(E\&T) , Aug.2017,Vol.32.

Fig. 5. The model of robot imported in Simulink 